\title{
HARMONIC MEASURE IN CONVEX DOMAINS
}

\author{
DAVID JERISON
}

Introduction. Let $\Omega$ be an open, convex subset of $\mathbf{R}^{N}$. At almost every point $x$ of $\partial \Omega$, with respect to surface measure $d \sigma$, there is a unique outer unit normal $\theta$. The map $g: \partial \Omega \rightarrow S^{n}$ given by $g(x)=\theta$ and defined almost everywhere is called the Gauss map. $\left(S^{n}, n=N-1\right.$, is the unit sphere in $\mathbf{R}^{N}$.) Suppose that the origin 0 belongs to $\Omega$. Harmonic measure for $\Omega$ at 0 is the probability measure $\omega$ such that for all continuous functions $f$ on $\partial \Omega^{1}$,

$$
u(0)=\int_{\partial \Omega} f d \omega
$$

where $u$ solves the Dirichlet problem: $\Delta u=0$ in $\Omega$ and $u=f$ on $\partial \Omega$.

Since $\Omega$ is a Lipschitz domain, Dahlberg's theorem [4] implies that $d \omega$ and $d \sigma$ are mutually absolutely continuous. Thus we can define a measure $\mu$ on $S^{n}$ by $\mu=g_{*} \omega$ or

$$
\mu(E)=\omega\left(g^{-1}(E)\right) \text { for all } E \subseteq S^{n} .
$$

We would like to pose the inverse problem: Given a probability measure $\mu$ on $S^{n}$, is there a domain $\Omega$ for which $\mu(E)=\omega\left(g^{-1}(E)\right)$ ? Loosely speaking, we would like to find the convex domain given harmonic measure as a function of the unit normal.

We will solve the problem in case $d \mu=R d \theta, R$ smooth and positive.

THEOREM 1. For $k$ an integer $\geq k(N)$ and $0<\alpha<1$, let $R \in C^{k, \alpha}\left(S^{N-1}\right)$ be a positive function with $\int R d \theta=1$. There exists a strictly convex domain $\Omega$ containing the origin, with $C^{k+2, \alpha}$ boundary, such that for $E \subset S^{N-1}$,

$$
\omega\left(g^{-1}(E)\right)=\int_{E} R d \theta,
$$

where $g$ is the Gauss map and $\omega$ is harmonic measure for $\Omega$ at 0 . The domain $\Omega$ is unique up to dilation.

Our problem is natural for three reasons. First, it is analogous and closely related to the classical Minkowski problem. Second it is entirely solved in the plane by a continuous version of the well-known SchwarzChristoffel formula. Third, the proof requires new, optimal estimates for

Received by the editors June 2, 1989.

1980 Mathematics Subject Classification (1985 Revision). Primary 30C35, 31B20, 35J60, 52A20, 53A05.

This research was supported in part by NSF grant DMS-8804582 and a Presidential Young Investigator Award. It was begun at the Mathematical Sciences Research Institute in Berkeley.

${ }^{1}$ If $\Omega$ is unbounded, we suppose further that $f$ tends to zero at infinity $\left(f \in C_{0}(\partial \Omega)\right)$ and that $u$ tends to zero at infinity. 
harmonic measure in convex domains. These estimates dovetail with recent optimal estimates for the Monge-Ampère equation due to L. Caffarelli. The proof of Theorem 1 depends by way of Caffarelli's methods on the entire development of regularity theory in the Minkowski problem. We would like to thank L. Caffarelli for explaining his recent results and for his encouragement.

The Minkowski problem is to find a convex body $\Omega$ given Gaussian curvature $K$ as a function of the unit normal. (See [3].) But $K$ is the Jacobian determinant of the Gauss map; informally, $d \theta=K d \sigma$ or $d \sigma=$ $(1 / K) d \theta$. Thus the Minkowski problem can be rephrased: Find the convex domain $\Omega$ given surface measure $d \sigma$ as a function of the unit normal. In our problem we have simply replaced surface measure with harmonic measure.

The Schwarz-Christoffel formula

$$
\boldsymbol{\Phi}^{\prime}(z)=r e^{i \psi} \prod_{k=1}^{m}\left(z-x_{k}\right)^{-\beta_{k}}, x_{1}<x_{2}<\cdots<x_{m}, \quad r>0, \psi \in \mathbf{R},
$$

gives the conformal mapping $\Phi$ from the upper half-plane $H=\{z \in$ C: $\operatorname{Im} z \geq 0\}$ to a polygon $\Omega$ with vertices $\Phi\left(x_{1}\right), \ldots, \Phi\left(x_{m}\right), \Phi(\infty)$ and exterior angles $\pi \beta_{1}, \ldots, \pi \beta_{m}, 2 \pi-\sum_{k=1}^{m} \pi \beta_{k}$. We confine ourselves to the convex case: $0<\beta_{k}<1$ and $\sum_{k=1}^{m} \beta_{k}<2$. (If $\sum_{k=1}^{m} \beta_{k} \leq 1$, then the polygon is unbounded, $\Phi(\infty)=\infty$.) Observe that $\arg \Phi^{\prime}(x)=x-\sum_{k=1}^{m} \pi \beta_{j}$ for $x \in\left(x_{k-1}, x_{k}\right) k=1, \ldots, m+1$, with the convention $x_{0}=-\infty, x_{m+1}=$ $\infty$. It follows that the outer normal to the side $\Phi\left(\left(x_{k-1}, x_{k}\right)\right)$ is $\alpha_{k}=$ $x-\pi / 2-\pi \sum_{j=k}^{m} \beta_{j}$. So far $\Omega$ is only defined up to translation. We can fix $\Phi$ by $\Phi(i)=0$. Let $c_{k}=\omega\left(\Phi\left(\left(x_{k-1}, x_{k}\right)\right)\right)$. Then $\mu=\sum_{c_{k}} \delta_{\alpha_{k}}$. Furthermore, since the harmonic measure for $\Omega$ at 0 is the push-forward of the harmonic measure for $H$ at $i, c_{k}=\frac{1}{\pi} \int_{x_{k-1}}^{x_{k}} d t /\left(1+t^{2}\right)$. Thus we can realize any $\mu$ that is a finite sum of delta functions by a suitable polygon $\Omega$.

In general, for an arbitrary probability measure $\mu$ on $[0,2 \pi)$ define the monotone function $U: \mathbf{R} \rightarrow[0,2 \pi)$ by

$$
U(x)=\min \left\{\alpha: \mu([0, \alpha]) \geq \frac{1}{\pi} \int_{-\infty}^{x} \frac{d t}{1+t^{2}}\right\}
$$

For any $E \subset[0,2 \pi), \mu(E)=\frac{1}{\pi} \int_{U^{-1}(E)} d t /\left(1+t^{2}\right)$. Define a conformal mapping $\Phi$ of $H$ by $\arg \Phi^{\prime}(x)-\pi / 2=U(x)$. Thus

$$
\Phi^{\prime}(z)=\exp [-V(z)+i(U(z)+\pi / 2)]
$$

where $V(z)$ is the harmonic conjugate of $U$. If we normalize $\Phi$ by $\Phi(i)=0$, then $g_{*}(\omega)=\mu$, as desired. Notice that $V$ is unique up to an additive constant, so that $\Omega$ is unique up to dilation. Regularity properties of the Poisson integral and Hilbert transform imply that if $d \mu=R d \theta$ with $R \in C^{k, \alpha}\left(S^{1}\right), k=0,1,2, \ldots 0<\alpha<1$ and $R>0$, then $U$ and $V$ belong to $C^{k+1, \alpha}$ and $\partial \Omega$ is a $C^{k+2, \alpha}$, strictly convex curve. 
Estimates for harmonic measure. The estimates for harmonic measure that we need are expressed in terms of cross-sections of $\Omega$. Let $H=\{x \in$ $\left.\mathbf{R}^{N}:\left(x-x_{0}\right) \cdot a \geq 0\right\}$ be a half-space. Let $E=\Omega \cap \partial H$ and $F=(\partial \Omega) \cap H$. Let $\frac{1}{2} E$ be the subset of $E$ given by dilation by the factor $1 / 2$ from an origin defined as the center of mass of $E$. Let $\frac{1}{2} F$ be the set of points of $F$ whose orthogonal projection onto $\partial H$ lies in $\frac{1}{2} E$.

THEOREM 2. Let $\Omega$ be a convex, open set in $\mathbf{R}^{N}$ such that $B_{r} \subset \Omega \subset B_{1}$. $\left(B_{r}\right.$ is the ball of radius $r$ about 0 .) There is a constant $C$ depending only on $N$ and $r$ such that

(a) $\omega(F) \leq C \omega\left(\frac{1}{2} F\right)$,

(b) $\max _{x \in F} h(x) \leq C \omega(F) / \sigma(F)$, where $h=d \omega / d \sigma$.

These estimates should be compared with the case of Lipschitz domains. (See, e.g. [5].) Estimate (b) permits $h$ to vanish but not to blow up. The key difference with the Lipschitz case is that instead of looking at "surface balls" of the form $B_{t}(x) \cap \partial \Omega$ we need to use the sets $F$, which resemble ellipses with uncontrolled eccentricity.

THEOREM 3. Suppose that $\Omega$ is a convex domain $B_{r} \subset \Omega \subset B_{1}, \partial \Omega$ is $C^{1}$ and strictly convex. For any $\delta>0$ there is $\varepsilon>0$ depending only on $\delta$, $N, r$, the $C^{1}$ modulus of continuity and the modulus of strict convexity such that

$$
\max _{x \in F} h \leq(1+\delta) \omega(F) / \sigma(F) \text { whenever } \sigma(F) \leq \varepsilon .
$$

This improvement of $(b)$ is analogous to the improvement of Dahlberg's theorem in [6].

The main ingredient in the proof of Theorem 2 beyond what is already known for Lipschitz domains is that Green's function is almost concave in the following sense:

Lemma. Let $G$ be Green's function for $\Omega$ with pole at $0 .(\Delta G=-\delta$, so that $G \geq 0$.) There is a constant $C=C(N, r)$ such that

$$
G(x)+G(y) \leq C G((x+y) / 2) \quad \text { for all } x, y \in \Omega \backslash B_{r} .
$$

Sketch of the Proof of Theorem 1 . We find $\Omega$ by the method of continuity. Let $\tau_{n}=\int_{S^{n}} d \theta$. Let $R^{t}=(1-t) \tau_{n}^{-1}+t R$. We wish to show that $T=\left\{t \in[0,1]\right.$ : there is a strictly convex, $C^{k+2, \alpha}$ domain $\Omega^{t}$ with $\left.d \mu^{t}=R_{t} d \theta\right\}$ is both open and closed in $[0,1]$. Since, $\Omega^{0}=B_{1}$ yields $d \mu^{0}=\tau_{n}^{-1} d \theta$, we have $0 \in T$, and it follows that $T=[0,1]$.

To show that $T$ is open, we express the equation in terms of the Minkowski support function $u(\theta)=x \cdot \theta\left(x=g^{-1}(\theta)\right)$ on the sphere $S^{n}$. The domain $\Omega$ can be recovered from $u$ by considering $F(r, \theta)=r u(\theta)$, a function in polar coordinates on $\mathbf{R}^{N} \cdot \nabla F$ is homogeneous of degree 0 and $\nabla F: S^{n} \rightarrow \partial \Omega$ is the inverse mapping of $g$. From now on we will regard functions and measures on $S^{n}$ and $\partial \Omega$ as identified via $g$ and $\nabla F$. If $u_{i j}=\nabla_{i j} u$ denote covariant derivatives of $u$ in an orthonormal frame on $S^{n}$, the Minkowski equation is (see [3])

$$
\operatorname{det}\left(u_{i j}+u \delta_{i j}\right)=\frac{1}{K}=\frac{d \sigma}{d \theta} .
$$


Since $h=d \omega / d \sigma$ and $R=d \omega / d \theta$, we have

$$
h \operatorname{det}\left(u_{i j}+u \delta_{i j}\right)=R \text {. }
$$

The linearized equation is

$$
\begin{aligned}
L v & \left.\equiv \frac{d}{d s} h_{u+s v} \operatorname{det}\left((u+s v)_{i j}+(u+s v) \delta_{i j}\right)\right|_{s=0} \\
& =\left(h c_{i j} v_{i}\right)_{j}-\frac{1}{K} \Lambda(h v),
\end{aligned}
$$

where $c_{i j}\left(u_{j k}+u \delta_{j k}\right)=(1 / K) \delta_{i k}$ defines $c_{i j}$ and $\Lambda$ is the Neumann operator, that is the operator taking a function on $\partial \Omega$ to the normal derivative of its harmonic extension.

$\int R d \theta=1$ implies $\int L v d \theta=0$ for all $v$ and hence $L^{*} 1=0$. By the theory of linear elliptic equations, the range of $L$ is the orthogonal complement of the null space of $L^{*}$. (This is the only place at which the requirement $k \geq k(N)$ plays a role.) Thus, by the implicit function theorem, in order to prove that $T$ is open we need only show that $L^{*} v=0$ implies $v$ is constant.

We will simultaneously find the null space of $L$ and $L^{*}$. Notice that $R$ does not change when $u$ is replaced by $u+s u$ since the corresponding region is the dilate $(1+s) \Omega$. Therefore, $L u=0$. Conversely, we have

Proposition 1. If $L^{*} v=0$ or if $L(v u)=0$, then $v$ is constant.

The proposition follows immediately from the formula

$$
\int_{S^{n}} v L(u v) d \theta=-\int_{S^{n}} h u c_{i j} v_{i} v_{j} d \theta-\int_{\Omega} \beta|\nabla \bar{v}|^{2}
$$

where $\bar{v}$ is the harmonic extension of $v$ to $\Omega$ and $\beta$ is the harmonic function in $\Omega$ with boundary values $u h$. Indeed, $L^{*} v=0$ and $L(v u)=0$ both imply that the left-hand side is zero. But the right-hand side only vanishes when $v$ is constant.

In addition to proving that $T$ is open, the proposition contains a uniqueness result: the only infinitesimal changes that preserve $R$ are dilations. This is known as infinitesimal rigidity.

The fact that $T$ is closed depends on a priori inequalities. We need to show that given a strictly convex, $C^{k+2, \alpha}$ domain $\Omega$, the $C^{k+2, \alpha}$ norm and modulus of strict continuity are controlled by the $C^{k, \alpha}$ norm and positive lower bound of $R$. Then a standard limiting argument shows that $T$ is closed.

First of all, we dilate $\Omega$ so that $B_{1}$ is the smallest ball containing $\Omega$.

Proposition 2. There exists $r>0$, depending only on the lower bound for $R$ such that $B_{r} \subset \Omega \subset B_{1}$.

This can be proved by an easy argument involving comparison with a hemisphere.

Consider a function $w: D \rightarrow \mathbf{R}, D \subset \mathbf{R}^{n}$, such that $\left\{\left(w(x), x_{1}, \ldots, x_{n}\right)\right.$ : $x \in D\}$ is a portion of $\partial \Omega$. Because $B_{r} \subset \Omega \subset B_{1},|\nabla w|$ is bounded 
above, that is, we have a priori bounds on the Lipschitz constant of $w$. The normal vector is $\theta=(-1, \nabla w) / \sqrt{1+|\nabla w|^{2}}$, so that

$$
d \theta / d \sigma=\left(1+|\nabla w|^{2}\right)^{-((n+2) / 2)} \operatorname{det}\left(w_{i j}\right) \quad\left(w_{i j}=\partial^{2} w / \partial x_{i} \partial x_{j}\right)
$$

and our equation is

$$
\operatorname{det}\left(w_{i j}\right)=\left(1+|\nabla w|^{2}\right)^{(n+2) / 2} h / R .
$$

Apart from the factor $h$, this is the equation of the Minkowski problem. $R$ is a smooth function of $\theta$, so the right-hand side involves only first derivatives of $w$. The function $h$ is the normal derivative of Green's function. As one can see from $\mathbf{R}^{2}$ and from the linearized operator $L$, the factor $h$ should be viewed as a nonlinear first order pseudodifferential operator on $w$. Its regularity properties are only slightly worse that those of $R$ and $1+|\nabla w|^{2}$. Our strategy is to derive some regularity for $h$ and deduce some further regularity for $w$ from the Monge-Ampere equation $(* *)$. Next, based on new estimates for $w$, we can improve our regularity estimates for $h$, and so on.

THEOREM 4. Let $B_{r} \subset \Omega \subset B_{1}, \Omega$ a smooth, strictly convex domain in $\mathbf{R}^{n+1}$. Suppose that, locally, $\partial \Omega$ is given by the graph of functions $w$ satisfying $\operatorname{det}\left(w_{i j}\right)=f$. Suppose also that

$$
\max _{x \in E} f(x) \leq C_{1} \int_{\frac{1}{2} E} f(x) d x / \operatorname{vol}(E)
$$

for all sets $E=\left\{x \in \mathbf{R}^{n}: w(x) \leq a \cdot x+b\right\}$. Then the $C^{1}$ modulus of continuity and modulus of strict convexity of $w$ depend only on $C_{1}, r$, and $n$.

Note that Theorem 2 implies that $F=\left(1+|\nabla w|^{2}\right)^{(n+2) / 2} h / R$ satisfies the hypothesis of Theorem 4 because $|\nabla w|$ is bounded above and $R$ is bounded above and below by positive constants. Theorem 4 follows from the method used by L. Caffarelli [1] to derive the same conclusion from the stronger hypothesis $C_{1}^{-1} \leq f \leq C_{1}$.

As a result of Theorem 4 we have control on the modulus of continuity of the Gauss map and hence of $\left(1+|\nabla w|^{2}\right)^{(n+2) / 2} / R$. An application of Theorem 3 implies that our function $f$ satisfies, in addition,

$$
\max _{x \in E} f(x) \leq(1+\delta) \int_{E} f(x) d x / \operatorname{vol}(E)
$$

with $\delta \rightarrow 0$ as $\operatorname{vol}(E) \rightarrow 0$.

THEOREM 5. If, in addition to the hypothesis of Theorem 4, we have $(\dagger)$, then the $L^{p}$ norm of $w_{i j}$ is bounded a priori for all $i, j=1, \ldots, n$ and any $p<\infty$. In particular, the $C^{1, \alpha}$ norm of $w$ is bounded a priori for any $\alpha<1$.

Theorem 5 follows from the method of another theorem of Caffarelli [2] with the same conclusion as Theorem 5, under the stronger hypothesis that $f$ is positive and continuous.

Once we have $C^{1, \alpha}$ control of $w$ it follows that $h$ has an a priori positive lower bound and $h$ belongs to $C^{\alpha}$. From this point on the regularity of 
$\left(1+|\nabla w|^{2}\right)^{(n+2) / 2} / R$ and $h$ are identical, and a somewhat more standard bootstrap argument leads to a priori bounds on the $C^{k+2, \alpha}$ norm of $w$. This concludes the proof of existence.

For uniqueness observe that local uniqueness implies that the map from $C^{k+z, \alpha}$ domains (modulo dilation) to densities $R$ in $C^{k, \alpha}$ is a local homeomorphism. The existence proof, local uniqueness, and a bifurcation argument yield a continuous right inverse to this map. It is then easy to check that the range of the inverse map is both closed and open, and hence the map is surjective. I am indebted to P. Pansu and B. E. J. Pahlberg for explaining this proof of uniqueness to me.

One may be able to pursue the analogy with the Minkowski problem further. We propose that $\int_{\partial \Omega^{t}} x \cdot \theta d \omega(x)$ is a concave function of $t$ if $\Omega^{t}=(1-t) \Omega^{0}+t \Omega^{1}$, and that this function is linear only when $\Omega^{1}$ is a dilate of $\Omega^{0}$. This is analogous to the Brunn-Minkowski inequality, which leads to uniqueness in the generalized Minkowski problem (see [3]).

\section{REFERENCES}

1. L. A. Caffarelli, A localization property of viscosity solutions to the Monge Ampère equation and their strict convexity, preprint.

2. _ Interior $W^{2, p}$ estimates for solutions of the Monge Ampère equation, Ann. of Math. (2) (to appear).

3. S.-Y. Cheng and S.-T. Yau, On the regularity of the solution of the $n$-dimensional Minkowski problem, Comm. Pure Appl. Math. 29 (1976), 495-516.

4. B. E. J. Dahlberg, Estimates for harmonic measure, Arch. Rational. Mech. Anal. 65 (1977), 275-288.

5. D. Jerison and C. E. Kenig, Boundary value problems of Lipschitz domains, Studies in Partial Differential Equations, M. A. A. Studies in Math., vol. 23 (W. Littman, ed.), pp. $1-68$.

6. _ - The logarithm of the Poisson kernel of a $C^{1}$ domain has vanishing mean oscillation, Trans. Amer. Math. Soc. 273 (1982), 781-794.

Department of Mathematics, Massachusetts Institute of Technology, CamBRIDGE, MASSACHUSETTS, 02139 